\title{
Liver Cancer Identification using Adaptive Neuro-Fuzzy Inference System
}

\author{
Marwa I.M. Obayya \\ Electronics and Communication Engineering \\ Department \\ Faculty of Engineering, Mansoura University \\ Mansoura, Egypt
}

\author{
Nihal F.F. Areed \\ Electronics and Communication Engineering \\ Department \\ Faculty of Engineering, Mansoura University \\ Mansoura, Egypt
}

\author{
Abdulhadi Omar Abdulhadi \\ Electronics and Communication Engineering Department \\ Faculty of Engineering, Mansoura University \\ Mansoura, Egypt
}

\begin{abstract}
This paper describes the application of adaptive neuro-fuzzy inference system (ANFIS) model for classification of liver tumor as benign or malignant by analyzing CT liver images. Decision making was performed in four stages: in the first stage, image is enhanced to improve its quality. In the second stage, the liver is extracted based on thresholding and boundary extraction algorithms. Then it is given as input to Fuzzy C-mean (FCM) clustering algorithm to segment it's inside tumor object. In the third stage, texture features and Discrete Wavelet Transformation features are extracted. In the fourth stage, the ANFIS classifier is trained by these extracted features using the backpropagation gradient descent method in combination with the least squares method. To evaluate the effect of each type of features on the tumor classification process, these two sets of features are trained separately to take the right decision to classify the liver tumor as malignant or benign. The performance of the proposed approach was tested and evaluated using a group of patient's CT images and the experimental results confirmed that the proposed approach has potential in identifying the tumor type.
\end{abstract}

\section{Keywords}

Liver Cancer, CT, Tumor, Feature Extraction, FCM, ANFIS.

\section{INTRODUCTION}

Liver tumor is one of the highest causes of death due to cancer [1] [2]. Liver cancer (Hepatocellular carcinoma HCC) is the sixth most common malignant tumor in the world and the third most common cause of death from cancer [3]. According to World Health Organization Statistics cancer is responsible for 7.6 million deaths worldwide annually. Deaths from cancer worldwide are projected to continue rising, with an estimated 13.1 million deaths in 2030 [4]. Tumor can be classified into two types, malignant and benign. The type which doesn't spread to other organs is called benign tumor. In the other hand, the malignant tumor may invade and spread to other organs.

In order to give effective treatment to patients, doctors will need to know the features of the tumor [5]. An early diagnosis of liver problems will increase patient's survival rate [6]. An accurate detection and proper segmentation of liver tumor from Computed Tomography (CT) image is of high significance especially for early detection and diagnoses of cancer [1]. In the past two decades, CT has been widely used as the accurate noninvasive imaging technology for liver cancer diagnosis [7].

Liver segmentation is the first important step in automatic diagnosis of liver cancer [8]. Liver segmentation of an image is essential task before extracting features of liver diseases and plays an important role in the research of liver cancer CAD [9] [10]. Feature extraction and representation is a crucial step for digital image processing.

Texture features can be employed to discriminate between different tumors. The texture features from CT images can be extracted directly from the spatial space or from a transformed space. Wavelet transform is a better tool for feature extraction from images [11]. It is necessary to detect and diagnose malignant tumors, so that early treatment can save many lives. Automatic identification of CT liver tumor image is a challenging task.

It is proven that the artificial neural networks (ANNs) have a greater predictive power which is used for diagnosis of diseases [12]. Fuzzy set theory plays an essential role in uncertainty decision making in medical applications. Neurofuzzy systems use ANNs theory in order to detect their properties by processing data samples. ANFIS is a specific approach in neuro-fuzzy development, which has shown significant results in modeling nonlinear functions which is implemented successfully as a classification tool in biomedical engineering [13] [14].

This work aims at developing a CAD system to identify the liver tumor as benign or malignant. In order to do that the ANFIS classifier is used.

The rest of this paper is organized as follows; Section 2 presents the related works. Section 3 explains the ANFIS classifier. Section 4 presents the data collection. Section 5 introduces the proposed Approach. Finally, section 6 and section 7 are illustrated the experimental results and the concluded remarks, respectively.

\section{RELATED WORKS IN TUMOR IDENTIFICATIN}

In the literature, there are some studies regarding of liver cancer identification. In [15], the hepatic tumor from the CT liver images is analyzed utilizing region growing technique, watershed method, texture features. These features are used to classify the tumor as benign or malignant using Support 
Vector Machine (SVM) approach. The obtained results showed that the performance of the system is more than $96 \%$ accuracy to classify tumors as benign or malignant. An automated CAD system utilizing contourlet transform based multi-resolution texture analysis for diagnosis of liver tumors is presented in [16]. The obtained results showed that the performance of the extracted features based upon contourlet transform outperformed that wavelet based features. For classification of benign and malignant tumors, based on contourlet texture features, the classifier achieves 94.3\% accuracy, while the highest rate achieved by wavelet coefficients is $88.6 \%$.

Another CAD system was proposed in [17], in order to classify two diffused liver diseases, steatosis and cirrhosis. First, the liver is extracted by using adaptive threshold and morphological processing. Second, in a transformation domain, some statistical features are extracted using the Orthogonal Wavelet Transform (OWT).Finally, the two-layer probabilistic $\mathrm{NN}$ was used as a classifier. The classification accuracy of $95 \%$ was achieved. The next CAD example was introduced in [18]. Their system was adapted to classify the liver tumors as malignant or benign. As texture parameters, only the normalized autocorrelation coefficients were used. The SVM classifier is employed and the obtained classification accuracy was about $82 \%$.

Recently, a texture-based CAD system is introduced in [19] to discriminate between malignant and benign liver tumors. Their work tested several sets of features: gray-level texture features, Wavelet Coefficient Texture (WCT) features, and Contourlet Coefficient Texture (CCT) features. The probabilistic Neural Network classifier is used. The highest classification accuracy was $96.7 \%$ were obtained with the contourlet coefficient co-occurrence features.

\section{ADAPTIVE NEURO-FUZZY INFERENCE SYSTEM (ANFIS)}

An ANFIS is a type of artificial neural network that is maps first order Takagi-Sugeno fuzzy inference system. This technique was originated by Jyh-Shing and Roger Jang in 1993[13]. The typical two fuzzy if-then rules to describe the ANFIS architecture can be expressed as follow:

Rule 1: if $x$ is $A_{l}$ and $y$ is $B_{l}$, then $f_{l}=p_{l} x+q_{l} y+r_{l}$

Rule 2: if $x$ is $A_{2}$ and $y$ is $B_{2}$, then $f_{2}=p_{1} x+q_{2} y+r_{2}$

Where, $x$ and $y$ : are the crisp inputs; $A_{i}$ and $B_{i}$ : are linguistic variables; $f_{i}$ : are the outputs; $p_{l}, q_{l}$ and $r_{l}$ : are the adaptive consequent parameters that are updated in the forward pass in the learning algorithm. Each of the $i^{\text {th }}$ node at layer 1 denoted as $O_{l, i}$.

\subsection{ANFIS Architecture}

ANFIS architecture is consists of five layers of nodes as shown in figure 1 . The detailed function of each layer is illustrated as follows.

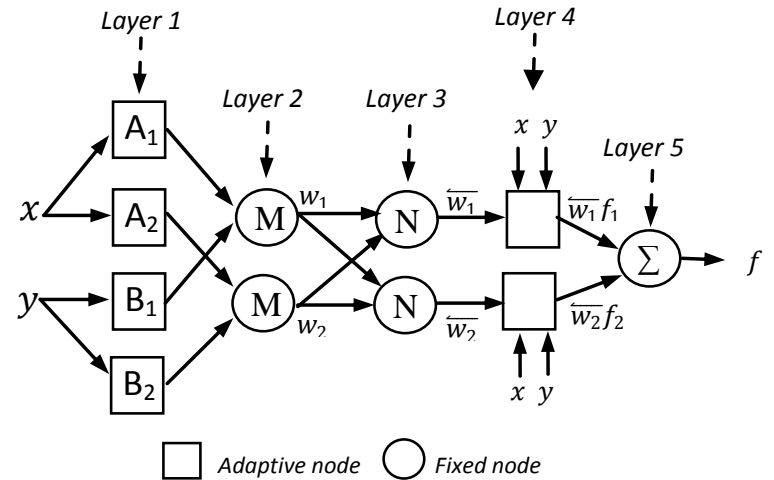

Fig 1: ANFIS architecture

In the first layer, each node in this layer is adaptive node. The outputs of this layer are the fuzzy membership grade of the inputs as follows.

$O_{1, i}=\mu_{A i}(x)$, for $i=1,2$

$O_{1, i}=\mu_{B i-2}(y)$, for $i=3,4$

Where, $\mu_{\mathrm{Ai}}(\mathrm{x}), \mu_{\mathrm{Bi}-2}(\mathrm{y})$ can adopt any fuzzy membership function.

A generalized bell membership function is selected due to the smoothness and concise notation. The bell membership function is specified by three parameters a, b, c. These parameters are referred to as premise parameters

$\mu_{A_{i}}(x)=\frac{1}{1+\left\{\left(\frac{x-c_{i}}{a_{i}}\right)^{2}\right\}^{b_{i}}}$

Where $a_{i}, b_{i}$ andc $c_{i}$ : the parameters of the membership function (MF).

In the second layer, each node is fixed node. Nodes are labeled with $\mathrm{M}$, indicating that they perform as a simple multiplier. The outputs of this layer can be formalized as:

$O_{2, i}=\omega_{i}=\mu_{A_{i}}(x) \mu_{B_{i}}(y), i=1,2$

Which are the so-called firing strengths of the rules.

In the third layer, the nodes are fixed and they are labeled with $\mathrm{N}$, indicating that they play a normalization role to the firing strengths from the previous layer. The outputs can be formalized as:

$O_{3, i}=\bar{\omega}_{i}=\frac{\omega_{i}}{\omega_{1}+\omega_{2}}, \quad i=1,2$

Which are the so-called normalized firing strengths.

In the fourth layer, the nodes are adaptive nodes. The output of each node is simply the product of the normalized strength and the first order Sugeno model as follows.

$O_{4, i}=\bar{\omega}_{i} f_{i}=\bar{\omega}_{i}\left(p_{i} x+q_{i} y+r_{i}\right), i=1,2$

The $\mathrm{p}, \mathrm{q}, \mathrm{r}$ parameters in this layer is referred to consequence parameters.

In the fifth layer, there is only one single fixed node labeled with S. This node performs the summation of all incoming signals. The overall output of the model is given by:

$O_{5, i}=\sum_{i} \bar{\omega}_{i} f_{i}=\frac{\sum_{i} \omega_{i} f_{i}}{\sum_{i} \omega_{i}}$ 


\subsection{Learning Algorithm of ANFIS}

The task of the learning algorithm for this architecture is to tune all the modifiable parameters, namely $\left\{a_{i}, b_{i}, c_{i}\right\}$ and $\left\{\mathrm{p}_{\mathrm{i}}, \mathrm{q}_{\mathrm{i}}, \mathrm{r}_{\mathrm{i}}\right\}$, to make the ANFIS output match the training data.

When the premise parameters $a_{i}, b_{i}$ and $c_{i}$ of the membership function are fixed, the output of the ANFIS model can be expressed as:

$$
\begin{gathered}
f=\left(\bar{\omega}_{1} x\right) p_{1}+\left(\bar{\omega}_{1} y\right) q_{1}+\left(\bar{\omega}_{1}\right) r_{1}+\left(\bar{\omega}_{2} x\right) p_{2} \\
+\left(\bar{\omega}_{2}\right) r_{2}
\end{gathered}+\left(\bar{\omega}_{2} y\right) q_{2}
$$

Which is a linear combination of the modifiable consequent parameters $\mathrm{p}_{1}, \mathrm{q}_{1}, \mathrm{r}_{1}, \mathrm{p}_{2}, \mathrm{q}_{2}$ and $\mathrm{r}_{2}$. The least squares method can be used to identify the optimal values of these parameters easily. A hybrid algorithm combining the least squares method and the gradient descent method is combined to train this model. The least squares method (forward pass) is used to optimize the consequent parameters with the premise parameters fixed. Once the optimal consequent parameters are found, the backward pass begins immediately. The gradient descent method (backward pass) is used to adjust optimally the premise parameters corresponding to the fuzzy sets in the input domain. The output of the ANFIS is calculated by employing the consequent parameters yielded in the forward pass. The output error is used to adapt the premise parameters by means of a standard backpropagation algorithm. It has been proven that this hybrid algorithm is highly efficient in training the ANFIS [13] [14].

\section{DATA COLLECTION}

To evaluate the proposed CAD system, a dataset of liver $C T$ were chosen from EL-Mogy Radiography Center at Mansoura University, the data sets contained a total of 100 images stored in JPEG format (among of them 50 cases with malignant liver tumors and the rest are 50 cases with benign liver tumors).

\section{PROPOSED METHODOLOGY}

The aim of this work is to present a CAD system to identify the liver tumor as benign or malignant. The proposed approach consists of four successive stages as shown in Figure 2.

Firstly, image enhancement stage, to improve the quality of the input image. The second stage, is image segmentation to extract the liver and tumor. The third stage is feature extraction and selection to extract the main features of the tumor object using special and transformation domains techniques. Finally, the ANFIS classifier is employed to classify the tumor as benign or malignant.

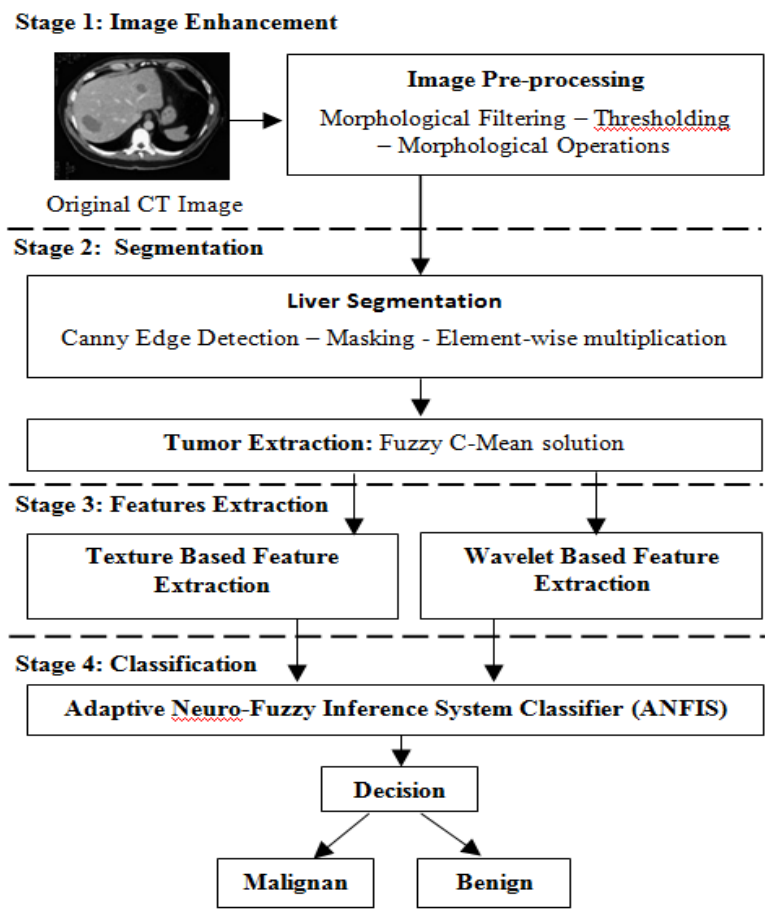

Fig.2 The proposed system block diagram

\subsection{Image Enhancement}

The original CT images of different scans and patients may have different contrast [20]. Thus morphological filter of mask $3 * 3$ is employed to remove noise.

\subsubsection{Morphological filtering}

Morphological filter is used for noise reduction as well as decoupling attached organs. For an input image (I), using a structuring element (B), the image is once dilated to become $I_{d i l}$, and once eroded to become $I_{\text {erod }}$. The average of both dilated and eroded images produces $I_{\text {avg }}$, as described in equations $(8,9)$.

$$
\begin{aligned}
& I_{\text {dil }}=I \oplus B \\
& I_{\text {erod }}=I \ominus B \\
& I_{\text {avg }}=\frac{I_{\text {dil }}+I_{\text {erod }}}{2}
\end{aligned}
$$

The new value assigned to the filtered image $\left(I_{\text {filt }}\right)$ at a certain location $(\mathrm{i}, \mathrm{j})$ is decided based upon the value of $I_{\text {avg }}$ at the same location, as described by equation (10).

$$
\begin{aligned}
I_{\text {filt }}(i, j)= & I_{\text {dil }} \text { if }(i, j) \geq I_{\text {avg }}(i, j) \\
& I_{\text {erod }} \text { if }(i, j)<I_{\text {avg }}(i, j)
\end{aligned}
$$

The input and output of the morphological filter is presented in the following figure 3.

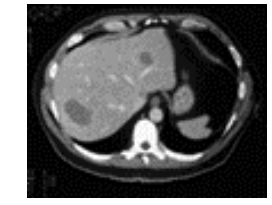

(a)

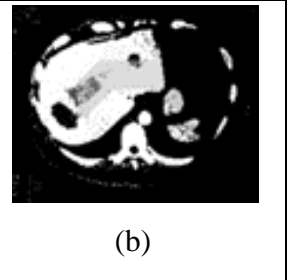

Fig 3: (a) Original input liver CT image. (b) Filtered image using $3 * 3$ morphological filter. 


\subsubsection{Phase-2: Image thresholding}

The output filtered image is then subjected to a thresholding process to be converted to binary image, in order to be further processed. As shown in figure 4, the thresholded image is then cleaned using morphological consecutive opening and closing processes. The main purpose of this cleaning process is to remove the small noise from the binary image. The connected component algorithm is implemented to find out the connected parts in this image. Then, the largest boundary is found.

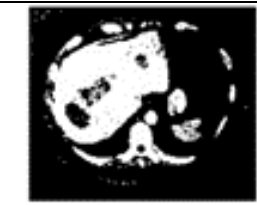

(a)

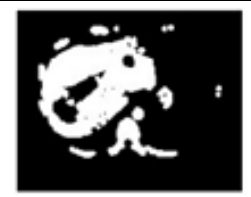

(b)
Fig 4: (a) Output of threshold process. (b) Cleaned threshold image.

\subsection{Liver and Tumor Segmentation}

Image segmentation is "the process of partitioning a digital image into multiple segments to simplify or change the representation of an image into something that is more meaningful" [21]. It is often a first step in a computer vision system for medical images diagnosis [8].

The ultimate aims of this stage are:

I) Segmenting the liver from the input CT image by separating it from other organs in the CT scan.

II) Segmenting the suspicious regions from the extracted liver. In order to do that we used the same methods in [22] which can be illustrated as follows:

\subsubsection{Liver Segmentation}

In multi-phases process, the liver is extracted via a set of preprocessing steps.

\subsubsection{Phase-1: Boundary extraction}

The boundaries of the detected components are extracted afterwards, then the largest boundary is extracted based upon the assumption that the liver is normally the largest organ in the input CT image.

\subsubsection{Phase-2: Masking}

Finally a mask is created by region filling algorithm to fill inside the largest boundary only.

\subsubsection{Phase-3: Element-wise multiplication}

By applying element-wise multiplication between the developed mask and the original input, the segmented liver can be obtained as shown in the following figure 6 .

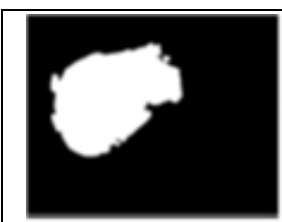

(a)

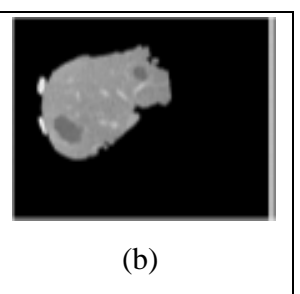

(b)
Fig 5: (a) Mask created, (b) Segmented liver image

\subsubsection{Tumor Extraction using FCM}

Clustering algorithms can be classified into hard clustering algorithms and soft clustering algorithms. Soft clustering algorithms associate an object to a particular cluster based on the degree of membership of this object to a particular cluster, using membership values to specify the convergence of an object to a cluster. Fuzzy C-means is a popular flat soft clustering algorithm, which is rather better than hard K-means [23]. In the proposed methodology, FCM clustering technique is used to segment the previously extracted liver into three clusters detecting the tumors as well. For each segmented liver image, FCM is applied to cluster its pixels into three different clusters i.e. background, liver and suspicious based on the intensity levels as shown in figure 6 . Based on the number of elements belonging to each cluster, an image containing the suspicious regions is detected. The small regions in this image are removed compared to the main suspicious regions, as they will be mainly noise or wrong pixels assigned to this cluster. Finally, only the suspicious region image is obtained as output of this stage.

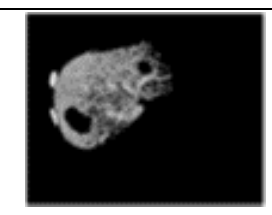

(a)

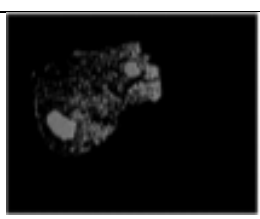

(b)
Fig 6: (a) Pixels belonging to liver cluster. (b) Pixels belonging to suspicious region cluster.

\subsection{Features Extraction}

Feature extraction is the operation to extract various image features for identifying or interpreting meaningful physical objects from image.

\subsubsection{Spatial Domain based Features Extraction} In this proposed work, the following 5 different texture features are considered [24].

1- GLCM (Gray Level Co-occurrence Matrix): is a statistical method of extracting textural features of an image. It is tabulation of how often the different combinations of pixel brightness value occur in an image.

2- Contrast: It measures the local variations in the gray-level co-occurrence matrix.

$$
\operatorname{CON}=\sum_{i, j \in G}(i-j)^{2} \cdot \operatorname{co}(i, j)
$$

3- Correlation: It measures the joint probability occurrence of the specified pixel pairs.

$$
\text { COR }=\sum_{i, j=0}^{G-1} P(i, j)\left(i-\mu_{i}\right)\left(j-\mu_{j}\right) / \sigma_{i} \sigma_{j}
$$

4- Energy: It provides the sum of squared elements in the gray-level co-occurrence matrix (GLCM), also known as uniformity or the angular second moment.

$$
A S M=\sum_{i, j \in G}[\operatorname{co}(i, j)]^{2}
$$

5- Homogeneity: It measures the closeness of distribution of elements in the gray-level co- 
occurrence matrix (GLCM) to the GLCM diagonal.

$$
H O M=\sum_{i, j} \frac{P(i, j)}{1+|i-j|}
$$

\subsubsection{Wavelet Based Feature Extraction}

Wavelet transform which provides representation of an image at various resolutions is a better tool for feature extraction from images [11]. 2D Discrete Wavelet Transform (2D DWT) is used in image processing as a powerful tool solving to image analysis, denoising, image segmentation and other. This technique decomposes the images into different frequency sub-bands components and then study each component with a resolution matched to its scale.

The DWT can be formalized as:

$\mathrm{W}[\mathrm{m}, \mathrm{n}]=\sum_{\mathrm{x}} \mathrm{f}[\mathrm{x}] \psi_{\mathrm{m}, \mathrm{n}}[\mathrm{x}]$

where, discretized scale and translation parameters are given by $\mathrm{a}=2^{\mathrm{j}}$ ve $\mathrm{b}=\mathrm{k} 2^{\mathrm{j}}(\mathrm{k}, \mathrm{j} \in \mathrm{Z})$. Then, wavelet basis function is written in as:

$\psi_{\mathrm{j}, \mathrm{k}}[\mathrm{x}]=2^{-\frac{\mathrm{j}}{2}} \psi\left(2^{\mathrm{j}} \mathrm{x}-\mathrm{k}\right)$

In two-dimensional transforms, the DWT is applied to each dimension separately. This yield a multiresolution decomposition of the image into four subbands called the approximation (low frequency component) and details (high frequency component). The approximation (A) indicates a low resolution of the original image. The detail coefficients are horizontal $(\mathrm{H})$, vertical $(\mathrm{V})$ and diagonal (D). Figure 3 presents process of CT liver cancer image being decomposed into approximate and detailed components. This process is repeated continuously putting the approximation (A) subimage through another set of low pass and high pass filters up to eight levels yielding 24 features for each input image as shown in the following figure 7 .

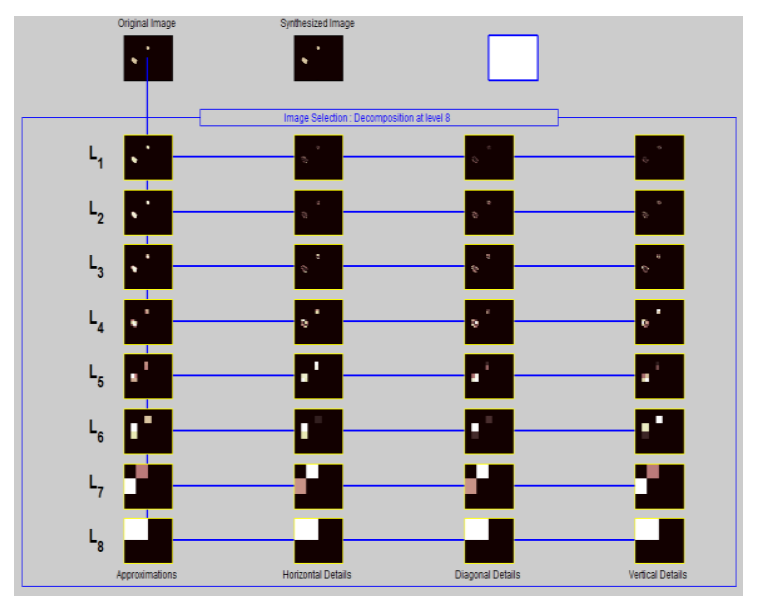

Fig. 7: Multi level Decomposition of CT Liver Cancer a) Approximation A1 b) Horizontal Detail H1 c) Vertical Detail V1 d) Diagonal Detail D1 e) Approximation A2 f) Horizontal Detail H2 g) Vertical Detail V2 h) Diagonal Detail D2.

here, the proposed approach is used this method to extract the wavelet features from The CT images of liver to help in the process of distinguishing between the malignant cancers and benign ones.

\subsection{Tumor Identification using ANFIS}

The proposed approach is used ANFIS for classification of $\mathrm{CT}$ liver tumors as malignant or benign.

The texture features and Discrete Wavelet Transformation features are extracted. ANFIS classifier is trained by these extracted features using the backpropagation gradient descent method in combination with the least squares method. To evaluate the effect of each type of features on the tumor classification process, these two sets of features are trained separately to take the right decision to classify the liver tumor as malignant or benign.

\section{EXPERIMENTAL RESUTS AND EVALUATION}

The proposed scheme is tested on 100 images stored in JPEG format (among of them 50 cases with malignant liver tumor and the rest are benign liver tumor). The output plots of the training and testing data obtained from ANFIS classifier are illustrated as shown in the following Figure 8.

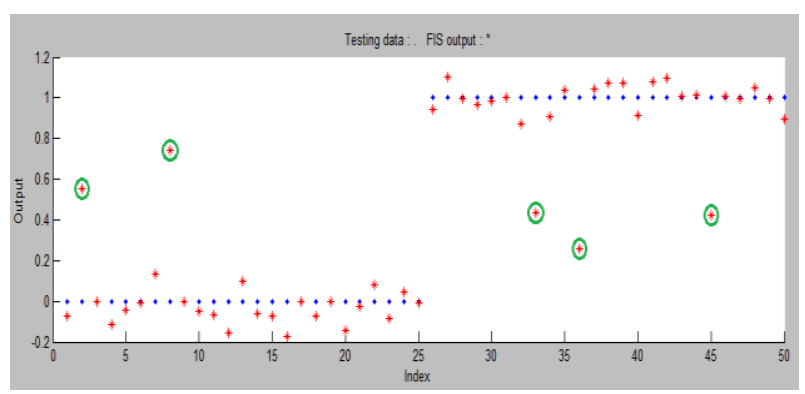

(a)

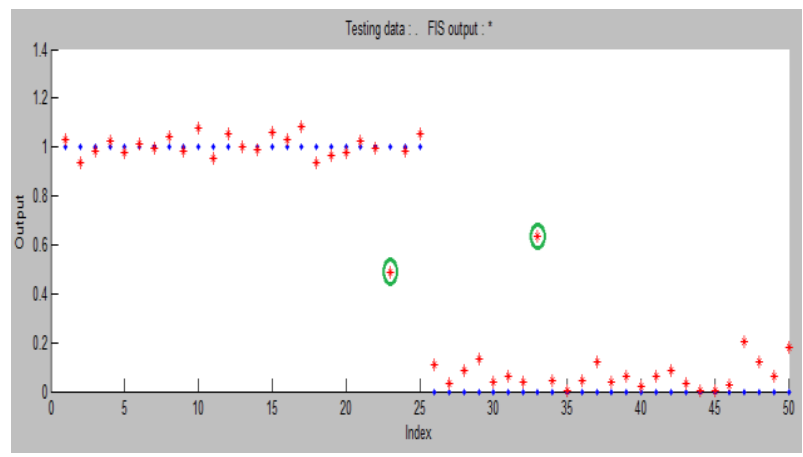

(b)

Fig. 8: (a) ANFIS output of training data against test data using texture features (b) ANFIS output of training data against test data using DWT based features

The texture features and DWT based features with the misclassified tumors enclosed in green circles. In texture based features Experiment, as shown in Table 1, of the 25 benign tumors, 23 were correctly identified as benign (TN), and two tumors were misclassified as malignant (FP). On the other hand, 22 tumors were correctly identified as malignant (TP) and only 3 tumors were misclassified as benign (FN). In DWT based features Experiment, as shown in Table.2, 24 tumors were correctly identified as benign (TN) and one tumor was misclassified as malignant (FP). On the other hand, 24 tumors were correctly identified as malignant (TP) and one tumor was misclassified as benign (FN).

To evaluate the diagnostic results, we used three measures named "Sensitivity", "Specificity" and "Accuracy" which are defined as follow: 


$$
\begin{aligned}
& \text { Sensitivity }=\frac{T P}{T P+F N} \\
& \text { Specificity }=\frac{T N}{T N+F P} \\
& \text { Accuracy }=\frac{T P+T N}{T P+F N+T N+F P}
\end{aligned}
$$

Where TP, TN, FN, FP stands for true positive, true negative, false positive and false negative, respectively.

TABLE 1. Confusion Matrix of Obtained Results from Test Set using Texture Based Features

\begin{tabular}{|c|c|c|}
\hline CAD System & Positive & Negative \\
\hline Positive (Malignant) & 22 & 3 \\
\hline Negative (Benign) & 2 & 23 \\
\hline
\end{tabular}

TABLE 2. Confusion Matrix of Obtained Results from Test Set using DWT based Features

\begin{tabular}{|c|c|c|}
\hline CAD System & Positive & Negative \\
\hline Positive (Malignant) & 24 & 1 \\
\hline Negative (Benign) & 1 & 24 \\
\hline
\end{tabular}

The performance of the proposed approach is presented in the following table 3 .

TABLE 3. Performance Measures of the Proposed System

\begin{tabular}{|c|c|c|c|}
\hline $\begin{array}{c}\text { Feature } \\
\text { Extraction } \\
\text { Technique }\end{array}$ & Accuracy & Sensitivity & Specificity \\
\hline Texture features & $90 \%$ & $91.6 \%$ & $90 \%$ \\
\hline DWT Features & $96 \%$ & $96 \%$ & $96 \%$ \\
\hline
\end{tabular}

\section{CONCLUDED REMARKS}

In this paper, the adaptive neuro-fuzzy inference system (ANFIS) classifier is trained by texture features and Discrete Wavelet Transformation features separately is proposed to classify CT tumor images into two classes that are malignant and benign. As the experimental results clearly show that the DWT is more effective than traditional texture features.

The logical next step in the future work is an intelligent advisory system to recommend appropriate treatment actions based on automated process to extract advanced tumor features and specify the type of the infection.

\section{REFERENCES}

[1] Weimin Huang; Ning Li; Ziping Lin; Guang-Bin Huang; Weiwei Zong; Jiayin Zhou; Yuping Duan, "Liver tumor detection and segmentation using kernel-based extreme learning machine," inEngineering in Medicine and Biology Society (EMBC), 2013 35th Annual International Conference of the IEEE, vol., no., pp.36623665, 3-7 July 2013

[2] Jie Lu; Defeng Wang; Lin Shi; Pheng Ann Heng, "Automatic liver segmentation in CT images based on Support Vector Machine," in Biomedical and Health Informatics (BHI), 2012 IEEE-EMBS International
Conference on , vol., no., pp.333-336, 5-7 Jan. 2012

[3] R. K. Bhullar and N. K.Walia. A New Hybrid Technique for Detection of Liver Cancer on ltrasound Images. International Journal of Science and Research (IJSR), Vol. 3,No. 10, pp. 1647- 1651, India,2014

[4] V.v.gomathi and s.karthikeyan. Performance evaluation of hmsk and sqfd algorithms for computer tomography (ct) image segmentation of effective radiotherapy. Journal of Theoretical and Applied Information Technology, Vol. 22,No. 2, pp. 1647- 1651, India,2014

[5] R.Rajagopal and P.Subbiah. Computer Aided Detection of Liver Tumor using SVM Classifier. International Journal of Advanced Research in Electrical, Electronics and Instrumentation Engineering, Vol. 3,No. 6, pp. 10170- 10177, India, June 2014

[6] B. V. Ramana1, Prof. M.Surendra Prasad Babu, and Prof. N. B. Venkateswarlu. A Critical Study of Selected Classification Algorithms for Liver Disease Diagnosis (IJAREEIE). International Journal of Database Management Systems ( IJDMS ), Vol. 3,No. 2, pp. 101114, India, May 2011

[7] Li Ma; Yang, L., "Liver Segmentation Based on Expectation Maximization and Morphological Filters in CT Images," in Bioinformatics and Biomedical Engineering, 2007. ICBBE 2007. The 1st International Conference on , vol., no., pp.690-693, 6-8 July 2007

[8] Yoshida, H.; Keserci, B.; Casalino, D.D.; Coskun, A.; Ozturk, O.; Savranlar, A., "Segmentation of liver tumors in ultrasound images based on scale-space analysis of the continuous wavelet transform," in Ultrasonics Symposium, 1998. Proceedings., 1998 IEEE, vol.2, no., pp.1713-1716 vol.2, 1998

[9] Zhiyuan Ma; Huiyan Jiang; Benqiang Yang; Zhang, L., "Unsupervised abdomen CT image segmentation using variable weight MRF in spatial and wavelet domain," in Computer Sciences and Convergence Information Technology (ICCIT), 2011 6th International Conference on , vol., no., pp.915-921, Nov. 29 2011-Dec. 12011

[10] Mala, K.; Sadasivam, V., "Automatic Segmentation and Classification of Diffused Liver Diseases using Wavelet Based Texture Analysis and Neural Network," in INDICON, 2005 Annual IEEE, vol., no., pp.216-219, 11-13 Dec. 2005

[11] P.Sinthia, Dr.K..Sujatha, M. Malathi. "Wavelet Based Decomposition and Approximation for Bone Cancer Image,". Australian Journal of Basic and Applied Sciences, March 2015, Pages: 344-350.

[12] Gu"ler I’, U” beyli ED. Detection of ophthalmic artery stenosis by leastmean squares backpropagation neural network. Comput Biol Med 2003;33(4):333-43.

[13] J.-S. R. Jang, "ANFIS: Adaptive-Network-based Fuzzy Inference Systems," IEEE Trans. on Systems, Man, and Cybernetics, vol. 23, pp. 665-685, May 1993.

[14] J.-S. R. Jang, C.-T. Sun, and E. Mizutani, "Neuro-Fuzzy and Soft Computing: A Computational Approach to Learning and Machine Intelligence," Prentice Hall, 1996.

[15] M. V. Sudhamani1 and G. T. Raju. Segmentation and Classification of Tumour in Computed Tomography Liver Images for Detection, Analysis and Preoperative 
Planning. International Journal of Advanced Computer Research, Vol. 4, N0. 1, pp. 166-171, Mar 2014

[16] S. S. Kumar, Dr. R.S. Moni, and J. Rajeesh. Contourlet Transform Based Computer-Aided Diagnosis System for Liver Tumors on Computed Tomography Images. International Conference on Signal Processing, Communication, Computing and Networking Technologies (ICSCCN 2011).

[17] Mala, K., Sadasivam, V.: Automatic segmentation and classification of diffused liver diseases using wavelet based texture analysis and Neural Network, Proc. of the Annual IEEE INDICON Conf., 2005, pp. 216-219.

[18] Huang, Y.L., Chen, J.H., Shen, W.C.: Diagnosis of hepatic tumors with texture analysis in nonenhanced computed tomography images, Acad. Radiol. 13(6), 2006, pp. 713-720.

[19] Kumar, S.S, Moni, R.S. Rajeesh, J.: An automatic computer-aided diagnosis system for liver tumours on computed tomography images, Comput. Electr. Eng. 39(5), 2013, pp. 1516-1526

[20] Weimin Huang; Yongzhong Yang; Zhiping Lin; GuangBin Huang; Jiayin Zhou; Yuping Duan; Wei Xiong, "Random feature subspace ensemble based Extreme
Learning Machine for liver tumor detection and segmentation," in Engineering in Medicine and Biology Society (EMBC), 2014 36th Annual International Conference of the IEEE, vol., no., pp.4675-4678, 26-30 Aug. 2014

[21] Elmasry, W.H.; Moftah, H.M.; El-Bendary, N.; Hassanien, A.E., "Performance evaluation of computed tomography liver image segmentation approaches," in Hybrid Intelligent Systems (HIS), 2012 12th International Conference on, vol., no., pp.109-114, 4-7 Dec. 2012

[22] M. Obayya and S.el.rabaie. Article: Automated Segmentation of Suspicious Regions in Liver CT using FCM. International Journal of Computer Applications 118(6):1-4, May 2015.

[23] W. M. Aly and H. A. Kelleny. Article: Adaptation of Cuckoo search for Documents Clustering. International Journal of Computer Applications 86(1):4-10, January 2014.

[24] AE Khedr, A Khalil, and MA Osman. Enhanced Liver Tumor Diagnosis Using Data Mining and Computed Tomography (CT). The international conference on Computing Technology and Information Management, Dubai, UAE, 2014. 